\title{
Optical Spectroscopy of V393 Scorpii During its Long Cycle
}

\author{
Ronald E. Mennickent ${ }^{1}$, Zbigniew Kołaczkowski ${ }^{2}$, Gojko Djurasevic ${ }^{3}$, \\ M. Diaz ${ }^{4}$ and Ewa Niemczura ${ }^{2}$ \\ ${ }^{1}$ Departamento de Astronomía, U. de Concepción, Casilla 160-C, Concepción, Chile \\ ${ }^{2}$ Instytut Astronomiczny Uniwersytetu Wrocławskiego, Wrocław, Poland \\ ${ }^{3}$ Astronomical Observatory, Volgina 7, 11060 Belgrade 38, Serbia \\ ${ }^{4}$ Departamento de Astronomia, IAG, U. de Sao Paulo, Rua do Matao, 1226, \\ Butanta 05508-900, Sao Paulo, SP, Brasil
}

\begin{abstract}
V393 Scorpii is a bright Galactic Double Periodic Variable showing a long photometric cycle of $\approx 253$ days. The ASAS V-band light curve has been disentangled into an orbital and long cycle component. The orbital light curve was modeled with two stellar components and a circumprimary accretion disk. Based on this model, and the careful choice of a template spectrum for the donor, the contribution of the donor to the line+continuum spectrum was removed at every orbital phase. The remaining residual spectra were analyzed. Notable findings are the larger line emissivity observed during the long cycle maximum that is concentrated to low velocities and the presence of discrete absorption components in the wings of the OI 7773 line, whose visibility strongly depends on the orbital phase. In addition, weak emission is observed in donor metallic absorption lines. Finally, we present the first $\mathrm{H} \alpha$ Doppler map for V 393 Scorpii. A modulated wind explains many observational features.
\end{abstract}

Keywords. stars: binaries, stars: early-type, stars: mass loss, stars: winds, outflows

This is a brief report on our recent investigation of the Double Periodic Variable (DPV) V393 Sco. DPVs are Algol-related binaries showing a long photometric periodicity besides the orbital photometric periodicity, they were first mentioned by Mennickent et al. (2003). The eclipsing binary V393 Sco resembles $\beta$ Lyrae in some aspects (Mennickent et al. 2010), but the amplitude of their long photometric cycle of 253 days is larger and the orbital light curves are more stable than in $\beta$ Lyrae. In V393 Sco, we observe in many lines the presence of discrete absorption components (DACs), mainly in the blue wing of the absorption lines, like in OI 7773 (Fig. 1). DACs are mostly visible during the second part of the orbital cycle and in the blue wing of the line. DACs are usually observed in the UV lines of some B-type stars and have been associated with condensations in an expanding stellar wind. Similarly, DACs in V393 Sco could be associated with an anisotropic wind emerging somewhere inside the gainer Roche lobe in the third or fourth quadrant.

We constructed $\mathrm{H} \beta$ difference spectra by subtracting a reference spectrum taken at similar orbital phase (first number in Fig. 2 labels) but different long phase (numbers in parentheses in same figure). We find that when the system goes to long cycle maximum, emission increases, especially at low velocities; similarly for $\mathrm{H} \alpha$ and $\mathrm{H} \gamma$. We interpret this as evidence for a polar wind whose modulation produces the long photometric cycle.

The Doppler map for the $\mathrm{H} \beta$ emission lines can be interpreted in terms of the existence of an optically thin polar wind plus a circumprimary optically thick region (Fig. 2).

In V393 Scorpii, the cooler stellar component is detected in hydrogen lines as well as in a characteristic A-type metallic spectrum. The new finding is the detection of emission 

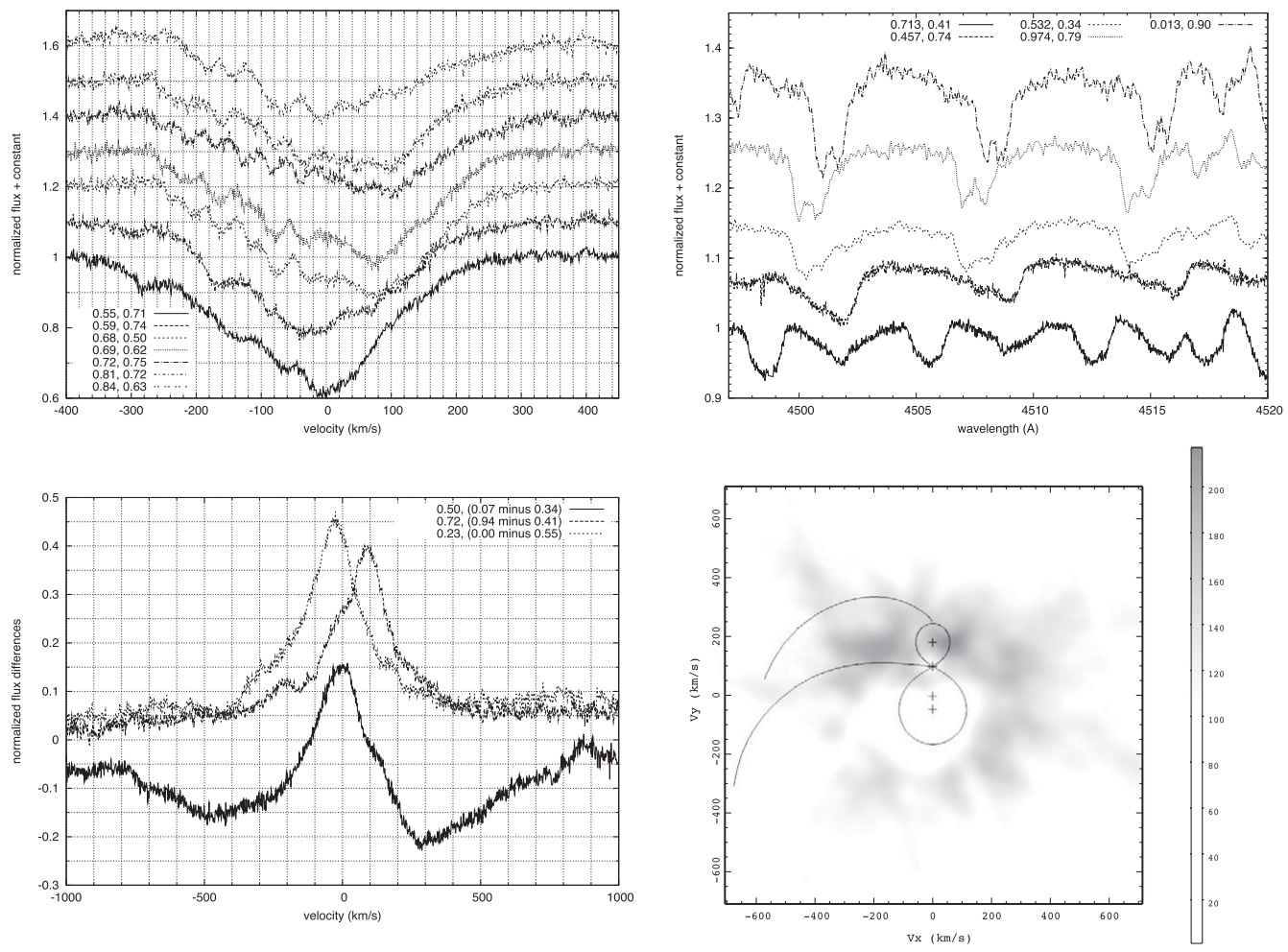

Figure 1. Top left: Example of DACs observed in O I 7773. Hereafter orbital (left) and long (right) phases are given. Top right: Example of emission cores, asymmetries and doubling of metallic lines. Bottom left: Difference spectra for $\mathrm{H} \beta$ emission lines at selected epochs. Velocities are with respect to the system center of mass. Bottom right: The back-projection Doppler map for the donor-subtracted $\mathrm{H} \alpha$ line during epochs of long cycle maximum $\left(0.8<\Phi_{l}<0.3\right)$. Crosses show, from up to down, donor center of mass, L1 point, system center of mass and gainer center of gravity. Roche lobe surfaces for a point mass gainer are shown along with the gas stream path (lower track). The upper track represents the keplerian velocity of a disk if it would exist along the stream.

lines in these optical metallic lines and the detection of an additional metallic spectrum arising from the accretion disk (Fig. 1). Emission cores are visible at main eclipse in the upper spectrum, and disk absorption components are clearly separated from the donor components in the bottom spectrum taken at quadrature.

After removing the donor spectrum by modeling its contribution at each orbital phase, we discovered at some epochs double emission lines in some iron, titanium and carbon lines (not shown here). We measured the radial velocity of these anomalous emissions observed in the metallic lines and found that they roughly follow the radial velocity path of the donor, suggesting an origin somewhere around the donor. The connection of these emission features with the long cycle will be explored in a forthcoming paper.

\section{References}

Mennickent, R. E., Pietrzynski, G., Diaz, M., \& Gieren, W. 2003, A\&SA 399, L47

Mennickent, R. E., Kołaczkowski, Z., Graczyk, D., \& Ojeda, B. 2010, MNRAS 405, 1947 